\title{
Does the U.S. exercise contagion on Italy? A theoretical model and empirical evidence
}

\author{
Roy Cerqueti ${ }^{a *}$ Livio Fenga $^{b}$, Marco Ventura $^{b}$ \\ ${ }^{a}$ Department of Economics and Law, University of Macerata, Italy \\ ${ }^{b}$ ISTAT, Italian National Institute of Statistics, Rome, Italy
}

December 28, 2017

\begin{abstract}
This paper deals with the theme of contagion in financial markets. At this aim, we develop a model based on Mixed Poisson Processes to describe the abnormal returns of financial markets of two considered countries. In so doing, the article defines the theoretical conditions to be satisfied in order to state that one of them - the so-called leader - exercises contagion on the others - the followers. Specifically, we employ an invariant probabilistic result stating that a suitable transformation of a Mixed Poisson Process is still a Mixed Poisson Process. The theoretical claim is validated by implementing an extensive simulation analysis grounded on empirical data. The countries considered are the U.S. (as the leader) and Italy (as the follower) and the period under scrutiny is very large, ranging from 1970 to 2014.
\end{abstract}

Keywords: Econophysics; financial markets; abnormal returns; contagion; Mixed Poisson Process.

\section{JEL Classification: C46; C65}

\section{Introduction}

Connections among international financial markets represent one of the most relevant theme in the macroeconomic and macrofinance context (Dornbusch et al, 2000; Van Rijckeghem and Weder, 2001; Forbes, 2012; Bellenzier et al., 2016 and the literature herein cited). Some countries seem to play a leading role - they are leaders - and their abnormal performances are likely to affect those of peripheral markets - the followers. When the shocks of a market affect some others, we are in presence of the so called contagion effect.

\footnotetext{
${ }^{*}$ Corresponding author: Roy Cerqueti, University of Macerata, Dep. Economics and Law, Via Crescimbeni 14, I-62100, Macerata, Italy. Tel.:+39 0733 2583246, fax: +39 07332583205 . Email: roy.cerqueti@unimc.it
} 
This paper deals with contagion among financial markets, exploring how the shocks of a leader market transpose themselves in shocks of a follower market. The topic is relevant because the availability of $a d$ hoc statistical methods for the extraction and description of the mechanisms of contagious can be very important. In fact, studies aimed at knowing with reasonable precision if and when contagion might occur, or at building possible scenarios through simulations, are vital steps not only for a better comprehension of the investigated system, but also in that they can be functional for the stability of financial markets.

We proceed in two different and complementary directions. First, we conceptualize a model of econophysics nature based on stochastic processes, and argue that this model can accommodate for contagion cases. In our experiments, we consider the U.S. as the leader and Italy as the follower. In so doing, we agree with the stylized financial facts that the behavior of U.S. market is able to drive - after a delay - the paths of the European ones (see e.g. Samarakoon, 2011).

In more details, our theoretical model captures the connotations and features of shocks. In particular, a shock in a financial market can be defined according to the presence of an abnormal return. Furthermore, the time at which the shocks occur do not follow regular and periodic paths, but rather they are random. A shock or, equivalently a jump - is then viewed as a couple of stochastic terms, one for the (abnormal) size of the return and the other for the random times of occurrence. Random times are modeled through a Mixed Poisson Process (MPP). Such a typology of processes represents an extension of the usual Poisson Processes with parameter $\lambda$, in that $\lambda$ is assumed to be random. MPP's seem to be particularly appropriate for our purpose, because they describe random arrivals whose time distance is captured by a random variable.

Several theoretical applications have been proposed in the literature on MPPs (see e.g. Cinlar, 1995, Grandell, 1997) but, to the best of our knowledge, this is the first attempt to investigate such a topic from an empirical point of view by examining financial contagion.

In the framework of MPP's, we adapt to our specific context an invariance result for which a suitable transformation of a MPP is still a MPP (see e.g. Foschi and Spizzichino, 2008). In particular, contagion can be described by introducing a random "small enough" delay - the meaning of the terms "small enough" will be clear soon - between the jumps of the leader market and those of the follower one. In so doing, it is theoretically shown that the assumption of MPP for modeling the shocks of the leader and of the follower fits with the presence of a contagion of the former country over the latter one.

The empirical experiments are based on the standardized, comparable time series of equity markets provided by the Morgan Stanley country Index (MSCI). The investigated period ranges from $1^{\text {st }}$ January 1970 to $27^{\text {th }}$ May 2014, on a daily basis. First of all, we validate the theoretical assumption that the data obey to a MPP law, by applying a Kolmogorov-Smirnov test to compare the empirical distributions of the data with the theoretical MPP one. Second, we empirically work out the random delay between the shocks occurring in the U.S. and in Italy and study these series. Afterwards, the analysis of the series of the delays provides also interesting informa- 
tion on the time in which contagion propagates. The series of the delays is also shown to exhibit some regularity properties in terms of the rank-size rule (for rank-size rules and applications, see e.g. Ausloos, 2014; Ausloos and Cerqueti, 2016; Axtell, 2001; Blasius and Tönjes, 2009; Cerqueti and Ausloos, 2015; Dimitrova and Ausloos, 2015; Fairthorne, 2005; Fujiwara, 2004; Herteliu et al., 2015; Ioannides and Overman, 2003; Ioannides and Skouras, 2013; Zanette, 2006; Zipf, 1935, 1949). In particular, delays have been ranked in decreasing order, from the largest to the smallest one, and the resulting rank-size plot is shown to be well-fitted by an exponential law. This evidence adds more on the conceptualization of the time propagation of the shocks, by giving information on how the delays distribute in terms of their size.

The novelties of the paper can be broken down as follows. First, we propose a conceptualization of the dynamics of the equity markets data through MPP's. In so doing, we offer a new vision of the financial markets in terms of the distribution of the dates of the abnormal returns. Second, we theoretically support the presence of contagion among countries by applying an invariance result on the MPP's. Third, we provide a validation of the theoretical results for the U.S. and Italy data over a very wide period - 44 years: from 1970 to 2014 -, hence obtaining evidence of the presence of contagion among such countries. Fourth, in the context of the empirical analysis, we discuss how contagion propagates by exploring the distribution of the delays between the leader market and the corresponding one of the follower country. Fifth, we provide a rank-size analysis of the series of the delays and obtain an excellent exponential fit. It is worth noting that the assessment of such a rank-size regularity has been rather neglected in the literature for the case of contagion and shock propagation.

The rest of the paper is organized as follows. After revising the literature on contagion in Section 2, Section 3 presents the theoretical model and the key invariance result. Section 4 is devoted to the empirical experiments: it contains the description of the data, the adopted methodologies, the obtained findings and related comments. The last section concludes and traces lines for future research.

\section{Review of the literature}

There are many definitions of the term contagion available in literature, according to the different perspectives it is considered from, Forbes (2012). Consequently, from a technical point of view, contagion can be studied under a variety of assumptions and quantitatively assessed through a wide range of mathematical tools. One of the oldest and perhaps most intuitive approach relies on probability theory. Shortly, probability models are employed to assess the likelihood for a given country to suffer from a crisis conditional on the occurrence of the same event elsewhere in the economy. On the one hand, probabilistic methods enjoy the properties of the tools of statistical decision theory. On the other hand, statistical tests suffer from limited power when endogenous relationships between variables involved in the analysis arise and limited robustness in case of omitted variables. As a result, the related outcomes can be severely biased and of little practical use.

During the nineties many procedures focused on the analysis of comovements, have 
been implemented. They focus on measuring comovements connections by means of the coefficient of correlation (e.g. Bordo and Murshid, 2001; Baig and Goldfajan, 1998; Calvo and Reinhart, 1996, Forbes and Rigobon, 2002). The rationale behind such methods is of empirical nature and rests on the evidence that market comovements become more apparent during periods of crisis. For instance, many studies show how various contagious effects, triggered by the 1994 Mexican crisis, can be associated with comovements induced as a result (Calvo et al, 1996; Frankel and Schmukler, 1996; Agénor et al 2004). Unfortunately, this approach is not without drawbacks, indeed the correlation coefficient during period of crisis is strongly upwardly biased due to heteroskedasticity in asset price movements (Rigobon, 1998; Fernández-Arias and Rigobon 1998, Rigobon, 2002). Furthermore, additional amounts of bias can be introduced by effects this approach is unable to account for - e.g. risk perception, change in the preference structures or of economic fundamentals. Thus, a step ahead in the analysis has been moved by the application of multivariate autoregressive schemes to study cross-country correlation structures, Boschi (2005) and the literature herein cited. In essence, the dynamic impact of random disturbances and of a set of endogenous variables are measured by an impulse-response function. Although this method is more robust to the problem of omitted factors, it may still suffers from heteroskedasticity problems in the returns.

As a response to these problems dynamic correlation-based approaches have been developed in the two-thousands. Among them, it is worth mentioning the one proposed by Zhao et al (2010), based on time varying coherence functions. It consists in a sequential computation of the squared correlation coefficient between pairs of values of the spectral densities computed at same frequencies. This technique has also been refined to capture comovements at different frequencies and their changes in synchronization (Imen and Rim, 2012; Celik, 2012). However, a maintained assumption of this models is normality, which is quite often violated.

Lastly, approaches exploiting either extreme values - i.e. based on statistical tests performed on the correlations of returns' tail values - or jumps in prices, are currently widely employed. These methods are essentially based on shock analysis, as they focus on the effects generated by one or more impacts on a specific markets generated by a different country. Their rationale is rather simple: only shock-related impacts, above a certain threshold, are analysed. Being robust against different distributional assumptions and independent from the functional form of the shock propagation mechanisms, they are widely adopted (Poon et al 2004, Aït-Sahalia et al, 2010).

The method proposed in the present paper is consistent with such a framework, and it is posited within this last strand of the literature, as it is designed to detect contagion assuming the occurrence of a jump in a main (leading) market which propagates across the system and hits one or more peripheral markets. The phenomenon of contagion is statistically conceived as a stochastic disturbance - with magnitude greater than a predefined threshold - and whose form is of an impulsive outlier. 


\section{Theoretical model}

Consider a filtered probability space $\left(\Omega, \mathcal{F}, \mathbb{F}=\left\{\mathcal{F}_{t}\right\}_{t \geq 0} \mathbb{P}\right)$, which contains all the stochastic processes that will be defined in this paper.

Denote as $\left\{x_{t}\right\}_{t \geq 0}$ and $\left\{y_{t}\right\}_{t \geq 0}$ the stochastic processes of the daily returns of the financial markets associated to two countries. We are interested in the definition and in the modeling of the jumps - or, shocks - in the markets.

A jump occurs when the financial market experiences an abnormal return. Formally, the return in $\left\{x_{t}\right\}_{t \geq 0}$ (in $\left\{y_{t}\right\}_{t \geq 0}$ ) is abnormal when its absolute value is above a prefixed and large threshold $\beta_{x}>0\left(\beta_{y}>0\right)$. This procedure is consistent with Mishkin and White (2003) who, in order to identify crashes, define them as stock index declines in excess of 20 percent over a 12 month moving window. Of course, as the wideness of $\beta$ increases, the number of returns to be defined "abnormal" decreases. Moreover, the selection of a too small value of $\beta$ does not lead to a proper definition of abnormal returns. For example, the value $\beta_{x}=\inf _{t \geq 0} \mathbb{E}\left[x_{t}\right]$ might include in the set labeled abnormal also some returns which are evidently in line with the standard behavior of the index.

By construction, the series of abnormal returns is a subseries of the whole series $\left\{x_{t}\right\}_{t \geq 0}$ and $\left\{y_{t}\right\}_{t \geq 0}$. Moreover, the time in which an abnormal return is observed cannot be determined a-priori.

Thus, the series of abnormal returns can be described through two point processes. The process of the abnormal returns contained in $\left\{x_{t}\right\}_{t \geq 0}$ is formalized as $\mathcal{J}=$ $\left\{\left(T_{i}, C_{i}\right)\right\}_{i \in \mathbf{N}}$, while for $\left\{y_{t}\right\}_{t \geq 0}$ we have $\mathcal{K}=\left\{\left(S_{i}, D_{i}\right)\right\}_{i \in \mathbf{N}}$, where $T$ 's and $S$ 's represent the stochastic times at which the shocks occur and the $C$ 's and $D$ 's are the corresponding abnormal returns, respectively. Specifically, for each $i \in \mathbb{N}$, the $i$-th jump of the process $\mathcal{J}(\mathcal{K})$ occurs at time $T_{i}\left(S_{i}\right)$ and with size $C_{i}\left(D_{i}\right)$.

In the context of contagion, the identification of a leader market and of a follower one is crucial. The spirit of such roles lies in the fact that the shocks occurring in the former generate shocks in the latter with a delay. Therefore, our theoretical model might be useful in describing a situation of contagion when considering $\mathcal{J}$ and $\mathcal{K}$ as the processes of the abnormal returns of the leader country and of the follower one, respectively.

To fix ideas, as we will see in the next section, the leader market is assumed to be the U.S. one while the role of follower is played by Italy.

In the present paper, we are interested only in the time component of the processes $\mathcal{J}$ and $\mathcal{K}$, and the size of the jumps are introduced for the sake of completeness. Indeed, the presence of a cause-effect relation between leader and follower can be measured only by searching for the presence of a small time-delay between the jumps in $\mathcal{J}$ and the ones in $\mathcal{K}$. Hence, it is not needed a deep exploration of the sizes of the jumps for evaluating the presence of contagion. However, the size of the jumps will drive the definition itself of the contagion, with the assessment of the abnormal returns (see the next section on this point).

The statement of specific assumptions on the temporal components of the point processes $\mathcal{J}$ and $\mathcal{K}$ leads to an interesting invariance result, already known in the litera- 
ture. We recall it here for the reader convenience:

Proposition 1. Assume that:

(i) the arrival times T's are described by a one-dimensional MPP;

(ii) there exists a sequence of i.i.d. random variables $\mathbf{W}=\left\{W_{i}\right\}_{i \in \mathbf{N}}$ independent of the process $\left\{T_{i}\right\}_{i \in \mathbf{N}}$ and such that $S_{i}=T_{i}+W_{i}$, for each $i \in \mathbb{N}$.

Then the process $\left\{T_{i}\right\}_{i \in \mathbf{N}}$ is an MPP.

For the proof, see e.g. Mirasol (1963) and Eick et al. (1993).

Proposition 1 has a meaning of paramount importance. Indeed, it states that a MPP can be obtained by transforming an original MPP through the application of suitably chosen random delays. Therefore, we can identify two processes: a leader process which is the independent one and is represented by the process $\mathcal{J}$ in our framework - and a follower - which is obtained by applying a random delay to the leader and is represented here by $\mathcal{K}$.

\section{Empirical experiments}

\subsection{The data}

We have taken the standardized, comparable time series on equity markets provided by the Morgan Stanley Country Index (MSCI) with daily frequency since the $1^{\text {st }}$ January 1970 up to the $27^{\text {th }}$ May 2014 for the U.S. and Italy. They represent the daily series of returns introduced in Section 3, namely - with a reasonable abuse of notation - the processes called $\left\{x_{t}\right\}_{t \geq 0}$ and $\left\{y_{t}\right\}_{t \geq 0}$, respectively.

The choice of these indices is motivated by the fact that they provide exhaustive equity market coverage as each country index is comprehensive of every listed security in that market. Besides, the indices are obtained by applying a consistent construction and maintenance methodology, which allows for meaningful global views and cross regional comparisons across all market capitalization size, sector and style segments and combinations. ${ }^{1}$

This "continuous" data have been treated to identify the abnormal returns. Specifically, we have considered a threshold $\beta>0$ and denoted as abnormal returns the values of the daily return whose absolute values are greater than $\beta$. The value of the threshold has been set at $\beta=3 \%$. This setting allows us to include in the set of the abnormal returns a sufficiently high number of elements and maintain, at the same time, a meaningful value for identifying the abnormal returns.

After the treatment of the data, we have found two sets of couples "size of returns - time of returns" which is properly included into the original ones. In particular, we have found $N_{U S}+1$ abnormal returns for the U.S. and $N_{I}+1$ for Italy, over the considered period. For ease of reference, we still adopt the notation used Section 3 the and collect such abnormal returns into the sets $\mathcal{J}=\left\{\left(T_{i}, C_{i}\right)\right\}_{i=1, \ldots, N_{U S}+1}$ for

\footnotetext{
${ }^{1}$ For further details on the MSCI see http://www.msci.com/products/indexes
} 
$\left\{x_{t}\right\}_{t \geq 0}$ and $\mathcal{K}=\left\{\left(S_{i}, D_{i}\right)\right\}_{i=1, \ldots, N_{I}+1}$ for $\left\{y_{t}\right\}_{t \geq 0}$.

Notice that the elements of $\mathcal{J}$ and $\mathcal{K}$ are not equally spaced in time, because abnormal returns occur with no periodicity. Moreover, the absolute values of the sizes of the abnormal returns are above the threshold $\beta$ but the exact value cannot be deterministically a-priori identified. Therefore, $\mathcal{J}$ and $\mathcal{K}$ can be theoretically modeled through point processes, whose time components behave as in Proposition 1.

\subsection{The methodology}

First, we empirically prove that the time components of the couples in $\mathcal{J}$ and $\mathcal{K}$, namely $\left\{T_{i}\right\}_{i=1, \ldots, N_{U S}+1}$ and $\left\{S_{i}\right\}_{i=1, \ldots, N_{I}+1}$, respectively, can be suitably modeled through two MPP's. At this aim, a Kolmogorov-Smirnov test is applied. Once the first stage is reached, we search for the random delay between the processes $\mathcal{J}$ and $\mathcal{K}$, whose existence is guaranteed by Proposition 1.

\subsection{Elaborations and discussion}

In the spirit of the Kolmogorov-Smirnov test, a possible way to check for the concordance of the data in $\left\{T_{i}\right\}_{i=1, \ldots, N_{U S}+1}$ and $\left\{S_{i}\right\}_{i=1, \ldots, N_{I}+1}$ with the MPP distribution consists in comparing their empirical distribution function with the MPP theoretical one.

To proceed, we recall the density function of a MPP and take $\mathcal{J}$ as reference process. Consider $t>0$ and define the variable $X_{t}^{\mathcal{J}}$ counting the number of jumps of the process $\mathcal{J}$ occurred up to time $t$. Of course, $X_{t}^{\mathcal{J}}$ is strongly connected to the process of the times of $\mathcal{J}$. We say that the T's follow a MPP if there exists a distribution function $U$ such that

$$
P\left(X_{t+\eta}-X_{t}=k\right)=\int_{0}^{+\infty} \frac{e^{-\lambda \eta}(\lambda \eta)^{k}}{k !} \mathrm{dU}(\lambda), \quad \forall \eta \geq 0, \mathrm{k}=0,1,2, \ldots
$$

Let us define the distribution function of the density in (1) as

$$
F_{\eta}(k)=P\left(X_{t+\eta}-X_{t} \leq k\right) \quad \forall \eta \geq 0, k=0,1,2, \ldots
$$

Now, consider the empirical distribution of the random times T's in the time interval $(t, t+\eta)$ as given by the available data, and denote its $k$-th element as $\tilde{F}_{\eta}(k)$, for $\eta \geq 0$ and $k=0,1,2, \ldots$.

The null-hypothesis $H_{0}$ of the Kolmogorov-Smirnov test is that $F \equiv \tilde{F}$, and it is not possible to reject it when there exists a distribution function $U$ such that

$$
\sup _{k \in \mathbb{N}}\left|F_{\eta}(k)-\tilde{F}_{\eta}(k)\right| \leq H_{n, \tilde{n}}(\alpha)=\sqrt{\frac{n+\tilde{n}}{n \tilde{n}}} h(\alpha),
$$

In plain English, it is not possible to reject $H_{0}$ whenever the distance between the theoretical and the empirical distribution function is less than a given quantity, $H$. Such a quantity is a function of the rejection level $\alpha$ and of the number of the elements 


\begin{tabular}{||c||c|c|c|c|c|c||}
\hline \hline$\alpha$ & 0.001 & 0.005 & 0.01 & 0.025 & 0.05 & 0.1 \\
\hline \hline$h(\alpha)$ & 1.95 & 1.73 & 1.63 & 1.48 & 1.36 & 1.22 \\
\hline US: $H_{n, \tilde{n}}(\alpha)$ & 0.248528 & 0.220489 & 0.207744 & 0.188626 & 0.173332 & 0.155489 \\
\hline IT: $H_{n, \tilde{n}}(\alpha)$ & 0.204164 & 0.18113 & 0.17066 & 0.154955 & 0.142391 & 0.127733 \\
\hline \hline
\end{tabular}

Table 1: Critical Kolmogorov-Smirnov values for different levels of $h(\alpha)$ for both countries. The variation of the values depends also on the different levels of $n$ for the considered countries.

of the "theoretical" and the empirical sample, $n$ and $\tilde{n}$, respectively. To compute the theoretical distribution, we conveniently truncate $\mathbb{N}$ - the variation set of $k$ - at the maximum number of jumps that could be empirically observed, $\bar{k}$. That is, given that the time elapsed between two jumps is measured in days, if the sum of all the intervals is $n$, and only one jump per day is allowed, then $\bar{k}=n$ days. For the U.S. case, we have $\bar{k}=n=8734$, which can be labeled as the time span of jumps, while $\tilde{n}=62$ can be labeled as the number of different delays empirically observed. For Italy, we have $\bar{k}=n=10822$ and $\tilde{n}=92$.

See Table 1 for the quantities of interest. To proceed, we have considered $\eta=0.001$ and normalized the entire period of analysis, so that one day corresponds to $1 / n$. The distribution $U$ has been selected discrete and such that $P(\lambda=l)=C(l+1)^{3}$, being $C$ the normalizing constant, for each $l$.

With this choice, the Kolmogorov-Smironov test is equal to:

$$
\left\{\begin{array}{l}
\sup _{k \in \mathbb{N}}\left|F_{\eta}(k)-\tilde{F}_{\eta}(k)\right|=0.12323, \quad \text { for the case of U.S.; } \\
\sup _{k \in \mathbb{N}}\left|F_{\eta}(k)-\tilde{F}_{\eta}(k)\right|=0.116875, \quad \text { for the case of Italy, }
\end{array}\right.
$$

pointing out that one cannot reject $H_{0}$ at each level of $\alpha$ for both countries, being the tabulated values always less than the critical threshold in Table 1 . This evidence entitles us, with a certain level of confidence, to treat the series as MPP, and to present the results arising from the empirical application of the theoretical results. After checking that both $\left\{T_{i}\right\}_{i \in \mathbb{N}}$ and $\left\{S_{i}\right\}_{i \in \mathbb{N}}$ are MPP, we are authorized to use Proposition 1 and guess the existence of the delays $\left\{W_{i}\right\}_{i \in \mathbb{N}}$.

A time interval is a delay if and only if it represents the difference between two consecutive jumps, the first one in the U.S. and the last in Italy. Moreover, we have derived the series of the delays from the empirical data under three alternative constraints: $(i)$ a time interval is a delay if it is no longer than 10 days; $(i)$ a time interval is a delay if it is no longer than 30 days; (iii) no constraints.

Table 2 contains the descriptive statistics of the series of the delays under the three constraints.

Some facts emerge: the variability in the data tends to increase with the magnitude of the delay considered. However, the recorded variations are more than proportional in the case of infinite delay. Such a behavior might reflect the additional amount of 


\begin{tabular}{|c||c|c|c|}
\hline Statistical indicator & Case $(i)$ & Case $($ ii $)$ & Case $($ iii $)$ \\
\hline \hline MEAN & 4.61 & 6.67 & 34.68 \\
VARIANCE & 8.81 & 23.85 & 2562.26 \\
STD DEV & 2.97 & 4.88 & 50.62 \\
COEFF VAR & 0.64 & 0.73 & 1.46 \\
MEDIAN & 4.5 & 6 & 8 \\
SKEWNESS & 0.28 & 0.66 & 0.71 \\
KURTOSIS & -1.25 & -0.54 & 1.87 \\
\hline
\end{tabular}

Table 2: Descriptive statistics of the delays in the three cases of constraints. Notice the high variability of the considered statistics with respect to the magnitude of the delay.

uncertainty associated with longer delays. While the coefficient of variation recorded for the first two cases of delay seems to be comparable (respectively amounting to $64 \%$ and $73 \%$ ), in the case of unbounded delay its value is about $146 \%$. In addition, the differences between mode and mean value are much more distant for the infinite delay than for the other two cases. Here, such differences suggest - without making any distributional assumptions - that the data are fairly symmetric in the first two cases. On the other hand, for the case infinite delay, the much larger value recorded for the mean, in comparison with the median, is an indication of right skewed distribution. The skewness coefficient supports this idea, in that in the case of infinite delay, shows a value 5 times bigger than the one recorded for the 10 days delay. Finally, the analysis of the values for the kurtosis, shows that the case somewhat closer to a data distribution matching a Gaussian curve is the 30 days delay (kurtosis $=-0.54$ ). The remaining cases go in opposite directions: a flatter distribution for the 10 days (kurtosis $=-1.25)$ and a leptokurtic one for the infinite delay (kurtosis=1.87).

The set of observed delays between the shocks propagated from the U.S. to Italy exhibits also some regularity properties, from a rank-size perspective.

Consider a ranking of the unbounded delays according to their size as follows: rank $=1$ stands for the highest value of delay (188, in our case) and then the ranking procedure goes on in decreasing order, so that rank $=47$ is associated to delay $=1$.

If one calibrates the parameters of an exponential function

$$
f(x)=a \cdot \exp (b x), \quad a, b \in \mathbb{R}
$$

then one obtains a visually appealing fit with $R^{2}=0.9862$ for $a=223.6$ and $b=$ -0.127 (see Figure 1). This suggests the presence of a clear rank-size relationship. Such a relation, applied in this specific context, is rather new in the literature, and will push us to further explorations in future research. In fact, rank-size laws have been found for many sets of data, but never for the delays in the context of contagion and shocks propagation.

PLEASE, INSERT FIGURE 1 HERE 
Caption: Delays ranked by decreasing order according to their magnitude with the corresponding exponential fit (see formula (4)).

\section{Concluding remarks}

This paper deals with the relevant theme of financial contagion under a theoretical and an empirical perspective. Specifically, we discuss the probabilistic conditions on the times of occurrence of the abnormal returns for the U.S. and Italy, and derive the presence of a idiosyncratic delay among them. We empirically explore such delays over a very rich dataset, whence providing information on their regularity. At this aim, a rank-size analysis is carried out and an exponential law seems to fit in an excellent manner the considered observations. Some future directions could be explored: first, it could be interesting to see whenever the contagion effect takes place in a more general context and over different datasets; second, the rank-size analysis suggests to go further in the investigation of the regularity of the data. To this purpose, the digits rules like the Benford law could be properly assessed, to add also to this type of empirical literature.

\section{References}

[1] Agénor PR, Aizenman J., Hoffmaister A.W., 2004. The credit crunch in east Asia: what can bank excess liquid assets tell us? Journal of International Money and Finance, 23(1): 27-49.

[2] Aït-Sahalia Y., Cacho-Diaz J., Laeven R.JA, 2010. Modeling financial contagion using mutually exciting jump processes. Journal of Financial Economics, 117(3): $585-606$.

[3] Ausloos M. 2014. Toward Fits to Scaling-Like Data, but with Inflection Points \& Generalized Lavalette Function. Journal of Applied Quantitative Methods, 9(2): $1-21$.

[4] Ausloos, M., Cerqueti, R., 2016. A universal rank-size law. PLoS ONE, 11(11), $\mathrm{e} 0166011$.

[5] Axtell, R.L. 2001. Zipf Distribution of U.S. Firm Sizes. Science, 293(5536): 18181820.

[6] Baig M.T., Goldfajn I., 1998. Financial market contagion in the Asian crisis. International Monetary Fund wp, No. 98/155.

[7] Bellenzier L., Andersen J.V., Rotundo G., 2016. Contagion in the world's stocks exchanges seen as set of coupled oscillators. Economic Modelling, 59: 224-236.

[8] Blasius, B., Tönjes, R., 2009, Zipf's law in the popularity distribution of chess openings. Physical Review Letters, 103(21), 218701. 
[9] Bordo M.D., Murshid A.P., 2001. Are financial crises becoming increasingly more contagious? what is the historical evidence on contagion? in Forbes K. and Claessens S., eds. International Financial Contagion: How It Spreads and How It Can be Stopped, New York, Kluwer Academic Publications, 367-406.

[10] Boschi M. 2005. International financial contagion: evidence from the Argentine crisis of 2001-2002. Applied Financial Economics, 15(3): 153-163.

[11] Camara A., Heston S., 2008. Closed-form option pricing formulas with extreme events. Journal of Futures Markets 28, 213-230.

[12] Calvo G.A., Leiderman L., Reinhart C.M., 1996. Inflows of capital to developing countries in the 1990s. Journal of Economic Perspectives, 10(2): 123-139.

[13] Calvo S.G., Reinhart C.M., 1996. Capital flows to Latin America: is there evidence of contagion effects?. In Private capital flows to emerging markets, Calvo G.A., Goldstein M., and Hochreiter E. eds., . Washington, DC, Institute for International Economics: 151-71.

[14] Celik S., 2012. The more contagion effect on emerging markets: The evidence of dcc-garch model. Economic Modelling, 29(5): 1946-1959.

[15] Cerqueti, R., Foschi, R., Spizzichino, F., 2009. A Spatial Mixed Poisson Framework for Combination of Excess of Loss and Proportional Reinsurance Contracts. Insurance: Mathematics \& Economics, 45: 59-64.

[16] Cerqueti, R., Ausloos, M., 2015. Evidence of Economic Regularities and Disparities of Italian Regions From Aggregated Tax Income Size Data. Physica A: Statistical Mechanics and its Applications 421(1): 187-207.

[17] Cinlar, E., 1995. An introduction to spatial queues. In: Advances in queueing Probability and Stochastics Series. CRC, Boca Raton: 103-118.

[18] Dimitrova, Z., Ausloos, M., 2015. Primacy analysis of the system of Bulgarian cities. Central European Journal of Physics 13: 218-225.

[19] Dornbusch R., Park Y.C., Claessens S., 2000. How it spreads and how it can be stopped. World Bank Research Observer, 15(2): 177-197.

[20] Eick S.G., Massey W.A., Whitt W., 1993. The physics of the Mt/G/1 queue. Operations Research 41, 731-742.

[21] Fairthorne, R.A., 2005. Empirical Hypberbolic Distributions (Bradford-ZipfMandelbrot) for Bibliometric Description and Prediction. Journal of Documentation, 61(2): 171-193.

[22] Fernández-Arias E.., Rigobon R., 1998. Financial contagion in emerging markets. Wanted: World financial stability. Inter-American Deveopment Bank Press, Washington D.C.. 
[23] Forbes K.J., 2012. The "Big C": Identifying and mitigating contagion. NBER wp, No 18465.

[24] Forbes K.J., Rigobon R., 2002. No contagion, only interdependence: measuring stock market comovements. Journal of Finance, 57(5): 2223-2261.

[25] Frankel J.A., Schmukler S.L., 1996. Crisis, contagion, and country funds: Effects on east Asia and Latin America. Pacific Basin Working Paper Series, WP No. PB96-04

[26] Foschi, R., Spizzichino, F., 2008. The role of the Order Statistic Property in Mixed Spatial Poisson Processes. In: Proceedings of the International Workshop on Applied Probability. Université de Technologie de Compiègne.

[27] Fujiwara Y., 2004. Zipf law in firms bankruptcy. Physica A: Statistical Mechanics and its Applications, 337(1-2): 219-230.

[28] Grandell J., 1997. Mixed Poisson Processes. Chapman \& Hall, London.

[29] Herteliu C., Ileanu B.V., Ausloos M., Rotundo G., 2015. Effect of religious rules on time of conception in Romania from 1905 to 2001. Human Reproduction, 30(9): 2202-2214.

[30] Imen G.M., Rim A., 2012. A dynamic analysis of financial contagion: the case of the subprime crisis. Journal of Business Studies Quarterly, 4(2): 11-27.

[31] Ioannides Y.M., Overman H.G., 2003. Zipf's law for cities: an empirical examination. Regional Science and Urban Economics 33(2): 127-137.

[32] Ioannides Y.M., Skouras S., 2013. US city size distribution: robustly Pareto, but only in the tail. Journal of Urban Economics, 73(1): 18-29.

[33] Mirasol N.M., 1963. The output of an M/G/1 queueing system is Poisson. Operations Research 11, 282-284.

[34] Mishkin F., White E.N., 2003. U.S. stock market crashes and their aftermath: Implications for monetary policy. In: Hunter W.C., Kaufmann G. G., Pomerleano M. (Eds.), Asset Price Bubbles: The Implications for Monetary Regulatory, and International Policies. The MIT Press, Cambridge, MA, pp. 5379

[35] Poon S., Rockinger M., Tawn J., 2004. Extreme value dependence in financial markets: Diagnostics, models, and financial implications. Review of Financial Studies, 17(2): 581-610.

[36] Rigobon R., 1998. Informational speculative attacks: good news is no news. Federal Reserve Board IF Seminar Paper.

[37] Rigobon R., 2002. The curse of non-investment grade countries. Journal of Development Economics, 69(2): 423-449. 
[38] Samarakoon L.P., 2011. Stock market interdependence, contagion, and the U.S. financial crisis: The case of emerging and frontier markets. Journal of International Financial Markets, Institutions and Money, 21(5): 724-742

[39] Serguieva A., Wu H., 2007. Computational intelligence in financial contagion analysis. In Seventh International Conference on Complex Systems, Boston, Massachusetts: 1-12.

[40] Van Rijckeghem C., Weder B., 2001. Sources of contagion: is it finance or trade?. Journal of International Economics, 54(2): 293-308.

[41] Zanette, D.H., 2006. Zipf's law and the creation of musical context. Musicae Scientiae, 10(1): 3-18.

[42] Zhao H., Lu S., Zou R., Ju K., Chon K.H., 2005. Estimation of time-varying coherence function using time-varying transfer functions. Annals of biomedical engineering, 33(11): 1582-1594.

[43] Zipf, G.K., 1935. The Psychobiology of Language, Houghton-Mifflin.

[44] Zipf, G.K., 1949. Human Behavior and the Principle of Least Effort. AddisonWesley Press 